Acta Crystallographica Section E

Structure Reports

Online

ISSN 1600-5368

\section{2-Aminocyclohexan-1-aminium thiocyanate}

\section{Halima F. Salem, Siti Aishah Hasbullah and Bohari M. Yamin*}

School of Chemical Sciences and Food Technology, Universiti Kebangsaan Malaysia, UKM 43500 Bangi Selangor, Malaysia

Correspondence e-mail: bohari@ukm.my

Received 25 April 2012; accepted 8 May 2012

Key indicators: single-crystal X-ray study; $T=298 \mathrm{~K}$; mean $\sigma(\mathrm{C}-\mathrm{C})=0.003 \AA$; $R$ factor $=0.045 ; w R$ factor $=0.112 ;$ data-to-parameter ratio $=16.9$.

The title compound, $\mathrm{C}_{6} \mathrm{H}_{15} \mathrm{~N}_{2}{ }^{+} \cdot \mathrm{NCS}^{-}$, was obtained unexpectedly from the reaction mixture of benzoyl chloride, ammonium thiocyanate and cyclohexane-1,2-diamine. The cyclohexane ring adopts a chair conformation. In the crystal, $\mathrm{N}-\mathrm{H} \cdots \mathrm{S}$ and $\mathrm{N}-\mathrm{H} \cdots \mathrm{N}$ interactions involving the thiocyanate anion and both the amine and the aminium $\mathrm{N}$ atoms link the molecules, forming two-dimensional networks parallel to $(001)$.

\section{Related literature}

For a description of the Cambridge Structural Database, see: Allen (2002). For related thiocyanate structures, see: Selvakumaran et al. (2011); Khawar Rauf et al. (2008).
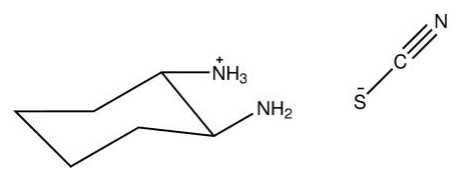

\section{Experimental}

\section{Crystal data}

$\mathrm{C}_{6} \mathrm{H}_{15} \mathrm{~N}_{2}^{+} \cdot \mathrm{NCS}^{-}$

$M_{r}=173.28$

Orthorhombic, $\mathrm{Pbca}$

$a=8.590(3) \AA$
Mo $K \alpha$ radiation
$\mu=0.29 \mathrm{~mm}^{-1}$

Data collection

Bruker SMART APEX CCD areadetector diffractometer

Absorption correction: multi-scan (SADABS; Bruker, 2000)

$T_{\text {min }}=0.870, T_{\max }=0.932$

Refinement

$R\left[F^{2}>2 \sigma\left(F^{2}\right)\right]=0.045$

$w R\left(F^{2}\right)=0.112$

$S=1.14$

1685 reflections
$T=298 \mathrm{~K}$

$0.50 \times 0.50 \times 0.25 \mathrm{~mm}$

10172 measured reflections 1685 independent reflections 1449 reflections with $I>2 \sigma(I)$ $R_{\text {int }}=0.028$

100 parameters

$\mathrm{H}$-atom parameters constrained

$\Delta \rho_{\max }=0.25{\mathrm{e} \AA^{-3}}^{-3}$

$\Delta \rho_{\min }=-0.16{\mathrm{e} \AA^{-3}}^{-3}$

Table 1

Hydrogen-bond geometry $\left(\AA,^{\circ}\right)$.

\begin{tabular}{lllll}
\hline$D-\mathrm{H} \cdots A$ & $D-\mathrm{H}$ & $\mathrm{H} \cdots A$ & $D \cdots A$ & $D-\mathrm{H} \cdots A$ \\
\hline $\mathrm{N} 1-\mathrm{H} 1 A \cdots \mathrm{S} 1^{\mathrm{i}}$ & 0.87 & 2.53 & $3.3914(19)$ & 172 \\
$\mathrm{~N} 1-\mathrm{H} 1 B \cdots \mathrm{N} 3^{\mathrm{ii}}$ & 0.82 & 2.10 & $2.895(3)$ & 166 \\
$\mathrm{~N} 1-\mathrm{H} 1 C \cdots \mathrm{N} 2^{\text {ii }}$ & 1.01 & 1.83 & $2.841(2)$ & 175 \\
$\mathrm{~N} 2-\mathrm{H} 2 A \cdots \mathrm{N} 3^{\mathrm{ii}}$ & 0.99 & 2.31 & $3.231(3)$ & 155 \\
$\mathrm{~N} 2-\mathrm{H} 2 B \cdots \mathrm{S} 1$ & 0.97 & 2.81 & $3.681(2)$ & 149 \\
\hline
\end{tabular}

Symmetry codes: (i) $x+1, y, z$; (ii) $-x+1,-y+1,-z$; (iii) $x+\frac{1}{2},-y+\frac{1}{2},-z$.

Data collection: SMART (Bruker,2000); cell refinement: SAINT (Bruker, 2000); data reduction: SAINT; program(s) used to solve structure: SHELXTL (Sheldrick, 2008); program(s) used to refine structure: SHELXTL; molecular graphics: PLATON (Spek, 2009); software used to prepare material for publication: SHELXTL, PARST (Nardelli, 1995) and PLATON.

The authors thank Universiti Kebangsaan Malaysia and the Ministry of Higher Education, Malaysia, for research grant No. UKM-GUP-NBT-08-27-110.

Supplementary data and figures for this paper are available from the IUCr electronic archives (Reference: LR2062).

\title{
References
}

Allen, F. H. (2002). Acta Cryst. B58, 380-388.

Bruker (2000). SADABS, SMART and SAINT. Bruker AXS Inc., Madison, Wisconsin, USA.

Khawar Rauf, M., Ebihara, M., Imtiaz-ud-Din, \& Badshah, A. (2008). Acta Cryst. E64, o366.

Nardelli, M. (1995). J. Appl. Cryst. 28, 659.

Selvakumaran, N., Karvembu, R., Ng, S. W. \& Tiekink, E. R. T. (2011). Acta Cryst. E67, o2843.

Sheldrick, G. M. (2008). Acta Cryst. A64, 112-122.

Spek, A. L. (2009). Acta Cryst. D65, 148-155. 


\section{supporting information}

Acta Cryst. (2012). E68, o1732 [doi:10.1107/S1600536812020879]

\section{2-Aminocyclohexan-1-aminium thiocyanate}

\section{Halima F. Salem, Siti Aishah Hasbullah and Bohari M. Yamin}

\section{S1. Comment}

The thiocyanate salts such as ammonium, potassium and sodium thiocyanate are useful reagents for organic synthesis specially for the formation of thiourea moiety. There are also some organic salts of thiocyanate such as dicyclohexylammonium thiocyanate which formed polymorph with orthorhombic (Khawar Rauf et al., 2008) and monoclinic (Selvakumaran et al., 2011) system respectively. Both salts were obtained rather unexpectedly from the mixture of benzoyl chloride, KSCN and dicyclohexylamine in the first and similarly, in the latter when isopthaloyl dichloride was used instead of benzoyl chloride. The title compound is analogous to the said compounds except the cation is a cyclohexane ring having a protonated and unprotonated amines at 1 and 2 positions respectively (Fig.1). The thiocyanate is linear with N3-C7-S1 bond angle of $178.22(19)^{\circ}$. The cyclohexane ring adopts a chair conformation. The bond lengths and angles are in normal ranges (Allen, 2002). In the crystal structure, the molecules are linked by N1-H1A $\cdots \mathrm{S} 1$, $\mathrm{N} 1-\mathrm{H} 1 \mathrm{~B} \cdots \mathrm{N} 3, \mathrm{~N} 1-\mathrm{H} 1 \mathrm{C} \cdots \mathrm{N} 2, \mathrm{~N} 2-\mathrm{H} 2 \mathrm{~A} \cdots \mathrm{N} 3$ and N2-H2B $\cdots \mathrm{S} 1$ intermolecular hydrogen bonds (symmetry codes as shown in Table 1) to form two-dimensional network (Fig. 2) parallel to (001).

\section{S2. Experimental}

All solvents and chemicals were of analytical grade and were used without purification. The mixture of benzoyl chloride $(1.41 \mathrm{~g}, 0.01 \mathrm{~mol})$, ammonium thiocyanate $(0.76 \mathrm{~g}, 0.01 \mathrm{~mol})$ and 1,2-diaminocyclohexane $(1.14 \mathrm{~g}, 0.01 \mathrm{~mol})$ in acetone was refluxed for $1 \mathrm{~h}$. After cooling the solution was filtered and left to evaporate at room temperature. Some good crystals were obtained after 5 days of evaporation. (Yield 82\%, m.p 395.9- 397.1 K). IR, NH: 3435.2, $3184.3 \mathrm{~cm}^{-1}, \mathrm{C}-\mathrm{N}$ -S: $2058 \mathrm{~cm}^{-1}, \mathrm{C}-\mathrm{N}: 1459 \mathrm{~cm}^{-1}$; CHNS, expt C: 48.22\%, N: 24.50\%, H: 8.73\%, S: 17.50\%), Calc C: 48.57, N: 24.20, H: 8.67, S: 18.49).

\section{S3. Refinement}

All $\mathrm{H}$ atoms attached to $\mathrm{C}$ atoms were fixed geometrically and treated as riding with $\mathrm{C}-\mathrm{H}=0.97 \AA$ (for $\left.\mathrm{CH}_{2}\right)$ and $0.98 \AA$ (for $\mathrm{CH}$ ) with $U_{\text {iso }}(\mathrm{H})=1.2 U_{\text {eq }}(\mathrm{C})$. The hydrogen atoms attached to nitrogen atoms were located from difference maps and refined using a riding model with $U_{\text {iso }}(\mathrm{H})=1.2 U_{\text {eq }}(\mathrm{N})$. 

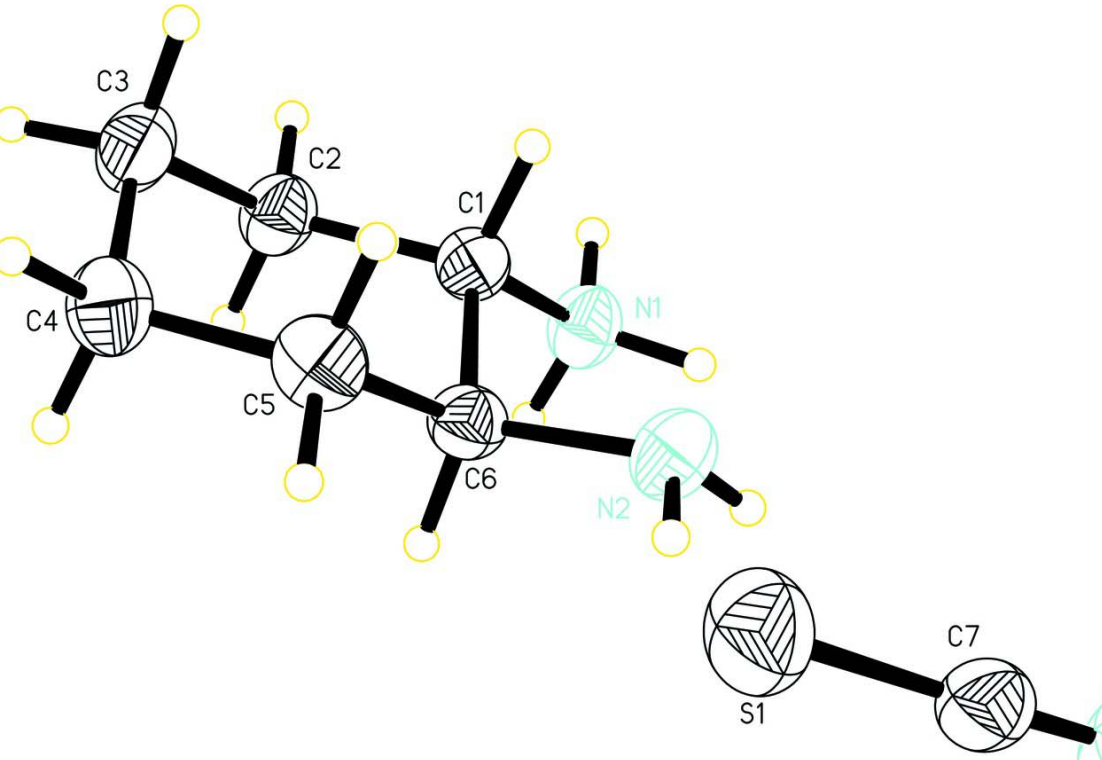

Figure 1

The molecular structure of (I), with displacement ellipsods drawn at the 50\% probability level.

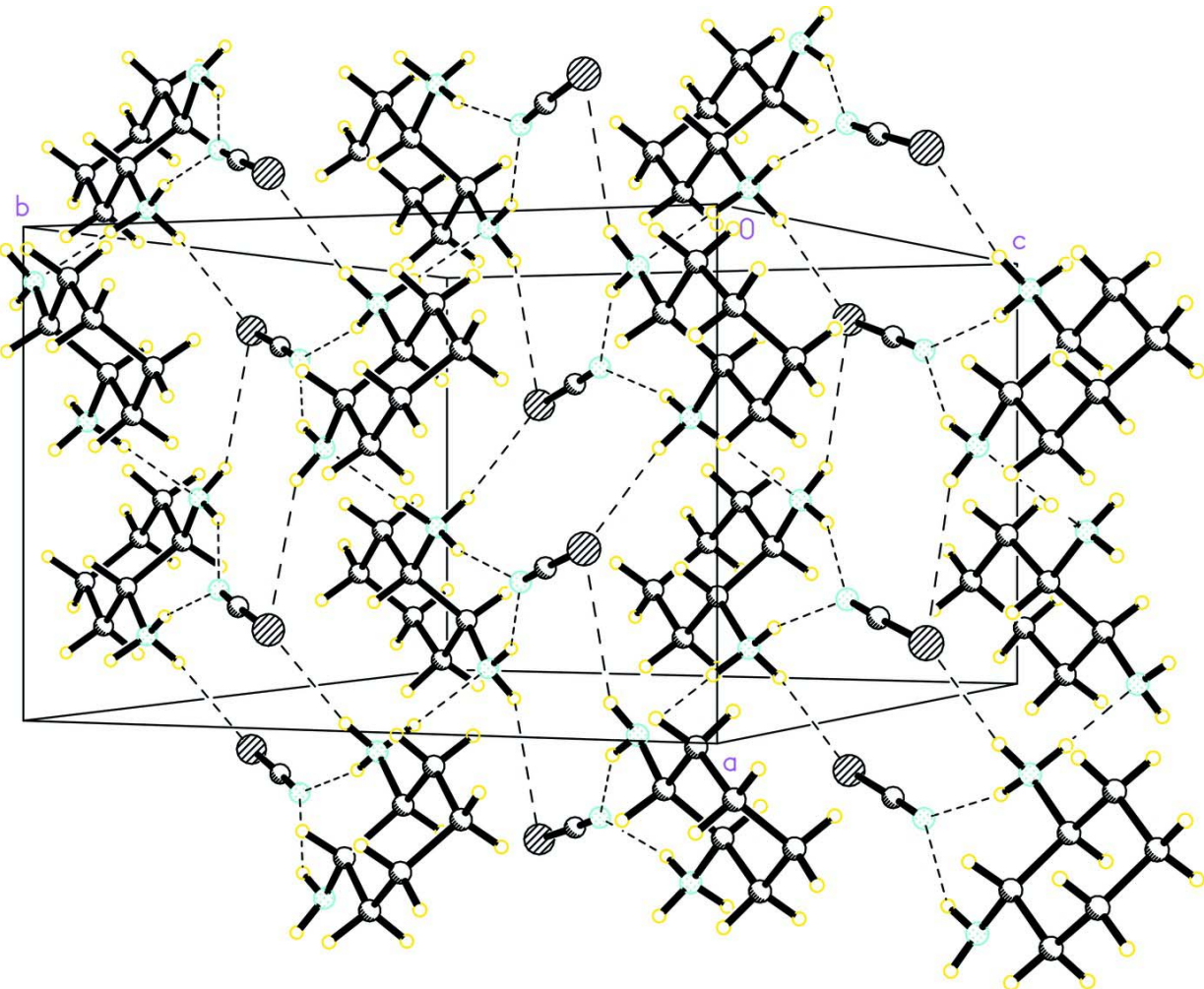

Figure 2

Molecular packing of (I) viewed down $c$ axis. The dashed lines indicate intermolecular hydrogen bonds. 


\section{2-Aminocyclohexan-1-aminium thiocyanate}

Crystal data

$\mathrm{C}_{6} \mathrm{H}_{15} \mathrm{~N}_{2}{ }^{+} \cdot \mathrm{NCS}^{-}$

$M_{r}=173.28$

Orthorhombic, $\mathrm{Pbca}$

Hall symbol: -P $2 \mathrm{ac} 2 \mathrm{ab}$

$a=8.590$ (3) $\AA$

$b=12.885(5) \AA$

$c=17.237$ (7) $\AA$

$V=1907.8(13) \AA^{3}$

$Z=8$

Data collection

Bruker SMART APEX CCD area-detector diffractometer

Radiation source: fine-focus sealed tube

Graphite monochromator

Detector resolution: 83.66 pixels $\mathrm{mm}^{-1}$

$\omega$ scan

Absorption correction: multi-scan

(SADABS; Bruker, 2000)

$T_{\text {min }}=0.870, T_{\max }=0.932$

Refinement

Refinement on $F^{2}$

Least-squares matrix: full

$R\left[F^{2}>2 \sigma\left(F^{2}\right)\right]=0.045$

$w R\left(F^{2}\right)=0.112$

$S=1.14$

1685 reflections

100 parameters

0 restraints

Primary atom site location: structure-invariant direct methods
$F(000)=752$

$D_{\mathrm{x}}=1.207 \mathrm{Mg} \mathrm{m}^{-3}$

Mo $K \alpha$ radiation, $\lambda=0.71073 \AA$

Cell parameters from 2210 reflections

$\theta=3.0-25.0^{\circ}$

$\mu=0.29 \mathrm{~mm}^{-1}$

$T=298 \mathrm{~K}$

Block, colourless

$0.50 \times 0.50 \times 0.25 \mathrm{~mm}$

10172 measured reflections

1685 independent reflections

1449 reflections with $I>2 \sigma(I)$

$R_{\text {int }}=0.028$

$\theta_{\max }=25.0^{\circ}, \theta_{\min }=3.0^{\circ}$

$h=-10 \rightarrow 10$

$k=-15 \rightarrow 12$

$l=-20 \rightarrow 18$

Secondary atom site location: difference Fourier map

Hydrogen site location: inferred from

neighbouring sites

$\mathrm{H}$-atom parameters constrained

$w=1 /\left[\sigma^{2}\left(F_{\mathrm{o}}^{2}\right)+(0.051 P)^{2}+0.6001 P\right]$

where $P=\left(F_{\mathrm{o}}^{2}+2 F_{\mathrm{c}}^{2}\right) / 3$

$(\Delta / \sigma)_{\max }=0.001$

$\Delta \rho_{\max }=0.25$ e $\AA^{-3}$

$\Delta \rho_{\min }=-0.16$ e $\AA^{-3}$

Special details

Geometry. All esds (except the esd in the dihedral angle between two 1.s. planes) are estimated using the full covariance matrix. The cell esds are taken into account individually in the estimation of esds in distances, angles and torsion angles; correlations between esds in cell parameters are only used when they are defined by crystal symmetry. An approximate (isotropic) treatment of cell esds is used for estimating esds involving 1.s. planes.

Refinement. Refinement of $F^{2}$ against ALL reflections. The weighted $R$-factor $w R$ and goodness of fit $S$ are based on $F^{2}$, conventional $R$-factors $R$ are based on $F$, with $F$ set to zero for negative $F^{2}$. The threshold expression of $F^{2}>\sigma\left(F^{2}\right)$ is used only for calculating $R$-factors(gt) etc. and is not relevant to the choice of reflections for refinement. $R$-factors based on $F^{2}$ are statistically about twice as large as those based on $F$, and $R$ - factors based on ALL data will be even larger.

Fractional atomic coordinates and isotropic or equivalent isotropic displacement parameters $\left(\AA^{2}\right)$

\begin{tabular}{lllll}
\hline & $x$ & $y$ & $z$ & $U_{\text {iso }} * / U_{\text {eq }}$ \\
\hline N1 & $0.87938(18)$ & $0.27887(13)$ & $0.03084(9)$ & $0.0437(4)$ \\
H1A & 0.9431 & 0.3073 & 0.0637 & $0.052^{*}$ \\
H1B & 0.8343 & 0.3242 & 0.0065 & $0.052^{*}$ \\
H1C & 0.9375 & 0.2357 & -0.0090 & $0.052^{*}$
\end{tabular}




$\begin{array}{lllll}\text { N2 } & 0.55776(18) & 0.33551(13) & 0.07851(9) & 0.0436(4) \\ \text { H2A } & 0.6130 & 0.3926 & 0.0516 & 0.052^{*} \\ \text { H2B } & 0.4757 & 0.3638 & 0.1112 & 0.052^{*} \\ \text { C1 } & 0.7706(2) & 0.20856(14) & 0.07357(10) & 0.0354(4) \\ \text { H1D } & 0.7077 & 0.1708 & 0.0355 & 0.043^{*} \\ \text { C2 } & 0.8674(2) & 0.13050(15) & 0.11858(12) & 0.0433(5) \\ \text { H2C } & 0.9300 & 0.0902 & 0.0827 & 0.052^{*} \\ \text { H2D } & 0.9374 & 0.1671 & 0.1532 & 0.052^{*} \\ \text { C3 } & 0.7648(3) & 0.05792(16) & 0.16548(12) & 0.0493(5) \\ \text { H3A } & 0.7022 & 0.0162 & 0.1305 & 0.059^{*} \\ \text { H3B } & 0.8297 & 0.0114 & 0.1957 & 0.059^{*} \\ \text { C4 } & 0.6591(3) & 0.11804(17) & 0.21932(12) & 0.0513(5) \\ \text { H4A } & 0.7213 & 0.1539 & 0.2579 & 0.062^{*} \\ \text { H4B } & 0.5904 & 0.0703 & 0.2462 & 0.062^{*} \\ \text { C5 } & 0.5629(2) & 0.19625(16) & 0.17426(11) & 0.0463(5) \\ \text { H5A } & 0.5010 & 0.2366 & 0.2104 & 0.056^{*} \\ \text { H5B } & 0.4919 & 0.1593 & 0.1403 & 0.056^{*} \\ \text { C6 } & 0.6618(2) & 0.26985(14) & 0.12574(10) & 0.0371(4) \\ \text { H6A } & 0.7235 & 0.3141 & 0.1603 & 0.045^{*} \\ \text { S1 } & 0.15812(8) & 0.37823(5) & 0.14873(3) & 0.0610(2) \\ \text { N3 } & 0.2362(3) & 0.53658(16) & 0.04577(11) & 0.0678(6) \\ \text { C7 } & 0.2023(2) & 0.47221(16) & 0.08883(11) & 0.0443(5)\end{array}$

Atomic displacement parameters $\left(\AA^{2}\right)$

\begin{tabular}{lllllll}
\hline & $U^{11}$ & $U^{22}$ & $U^{33}$ & $U^{12}$ & $U^{13}$ & $U^{23}$ \\
\hline $\mathrm{N} 1$ & $0.0428(9)$ & $0.0436(9)$ & $0.0446(9)$ & $0.0029(7)$ & $0.0051(7)$ & $0.0088(7)$ \\
$\mathrm{N} 2$ & $0.0366(8)$ & $0.0421(9)$ & $0.0520(10)$ & $0.0063(7)$ & $0.0000(7)$ & $0.0027(7)$ \\
$\mathrm{C} 1$ & $0.0349(9)$ & $0.0353(10)$ & $0.0361(9)$ & $-0.0039(8)$ & $0.0002(7)$ & $0.0009(8)$ \\
$\mathrm{C} 2$ & $0.0421(11)$ & $0.0409(11)$ & $0.0469(11)$ & $0.0055(9)$ & $0.0019(9)$ & $0.0032(9)$ \\
$\mathrm{C} 3$ & $0.0586(13)$ & $0.0400(11)$ & $0.0493(11)$ & $0.0026(10)$ & $0.0020(10)$ & $0.0085(9)$ \\
$\mathrm{C} 4$ & $0.0582(13)$ & $0.0522(13)$ & $0.0435(11)$ & $-0.0042(10)$ & $0.0079(10)$ & $0.0075(9)$ \\
$\mathrm{C} 5$ & $0.0408(10)$ & $0.0531(12)$ & $0.0451(10)$ & $-0.0008(9)$ & $0.0078(8)$ & $-0.0006(9)$ \\
$\mathrm{C} 6$ & $0.0366(10)$ & $0.0366(10)$ & $0.0383(9)$ & $-0.0002(8)$ & $-0.0025(8)$ & $-0.0029(8)$ \\
S1 & $0.0708(4)$ & $0.0589(4)$ & $0.0535(4)$ & $-0.0088(3)$ & $0.0002(3)$ & $0.0116(3)$ \\
$\mathrm{N} 3$ & $0.0815(15)$ & $0.0521(12)$ & $0.0697(12)$ & $0.0012(11)$ & $0.0043(11)$ & $0.0167(11)$ \\
$\mathrm{C} 7$ & $0.0420(11)$ & $0.0442(12)$ & $0.0467(11)$ & $0.0070(9)$ & $-0.0023(9)$ & $-0.0063(10)$ \\
\hline
\end{tabular}

Geometric parameters $\left(A,{ }^{\circ}\right)$

\begin{tabular}{llll}
\hline $\mathrm{N} 1-\mathrm{C} 1$ & $1.495(2)$ & $\mathrm{C} 3-\mathrm{C} 4$ & $1.512(3)$ \\
$\mathrm{N} 1-\mathrm{H} 1 \mathrm{~A}$ & 0.8682 & $\mathrm{C} 3-\mathrm{H} 3 \mathrm{~A}$ & 0.9700 \\
$\mathrm{~N} 1-\mathrm{H} 1 \mathrm{~B}$ & 0.8173 & $\mathrm{C} 3-\mathrm{H} 3 \mathrm{~B}$ & 0.9700 \\
$\mathrm{~N} 1-\mathrm{H} 1 \mathrm{C}$ & 1.0147 & $\mathrm{C} 4-\mathrm{C} 5$ & $1.517(3)$ \\
$\mathrm{N} 2-\mathrm{C} 6$ & $1.475(2)$ & $\mathrm{C} 4-\mathrm{H} 4 \mathrm{~A}$ & 0.9700 \\
$\mathrm{~N} 2-\mathrm{H} 2 \mathrm{~A}$ & 0.9911 & $\mathrm{C} 4-\mathrm{H} 4 \mathrm{~B}$ & 0.9700 \\
$\mathrm{~N} 2-\mathrm{H} 2 \mathrm{~B}$ & 0.9740 & $\mathrm{C} 5-\mathrm{C} 6$ & $1.523(3)$ \\
$\mathrm{C} 1-\mathrm{C} 2$ & $1.518(3)$ & $\mathrm{C} 5-\mathrm{H} 5 \mathrm{~A}$ & 0.9700
\end{tabular}




\begin{tabular}{|c|c|c|c|}
\hline $\mathrm{C} 1-\mathrm{C} 6$ & $1.519(2)$ & $\mathrm{C} 5-\mathrm{H} 5 \mathrm{~B}$ & 0.9700 \\
\hline $\mathrm{C} 1-\mathrm{H} 1 \mathrm{D}$ & 0.9800 & C6-H6A & 0.9800 \\
\hline $\mathrm{C} 2-\mathrm{C} 3$ & $1.518(3)$ & $\mathrm{S} 1-\mathrm{C} 7$ & $1.636(2)$ \\
\hline $\mathrm{C} 2-\mathrm{H} 2 \mathrm{C}$ & 0.9700 & $\mathrm{~N} 3-\mathrm{C} 7$ & $1.150(3)$ \\
\hline $\mathrm{C} 2-\mathrm{H} 2 \mathrm{D}$ & 0.9700 & & \\
\hline $\mathrm{C} 1-\mathrm{N} 1-\mathrm{H} 1 \mathrm{~A}$ & 109.2 & $\mathrm{C} 2-\mathrm{C} 3-\mathrm{H} 3 \mathrm{~A}$ & 109.4 \\
\hline $\mathrm{C} 1-\mathrm{N} 1-\mathrm{H} 1 \mathrm{~B}$ & 112.9 & $\mathrm{C} 4-\mathrm{C} 3-\mathrm{H} 3 \mathrm{~B}$ & 109.4 \\
\hline $\mathrm{H} 1 \mathrm{~A}-\mathrm{N} 1-\mathrm{H} 1 \mathrm{~B}$ & 109.4 & $\mathrm{C} 2-\mathrm{C} 3-\mathrm{H} 3 \mathrm{~B}$ & 109.4 \\
\hline $\mathrm{C} 1-\mathrm{N} 1-\mathrm{H} 1 \mathrm{C}$ & 108.0 & $\mathrm{H} 3 \mathrm{~A}-\mathrm{C} 3-\mathrm{H} 3 \mathrm{~B}$ & 108.0 \\
\hline $\mathrm{H} 1 \mathrm{~A}-\mathrm{N} 1-\mathrm{H} 1 \mathrm{C}$ & 111.2 & $\mathrm{C} 3-\mathrm{C} 4-\mathrm{C} 5$ & $110.68(17)$ \\
\hline $\mathrm{H} 1 \mathrm{~B}-\mathrm{N} 1-\mathrm{H} 1 \mathrm{C}$ & 106.1 & $\mathrm{C} 3-\mathrm{C} 4-\mathrm{H} 4 \mathrm{~A}$ & 109.5 \\
\hline $\mathrm{C} 6-\mathrm{N} 2-\mathrm{H} 2 \mathrm{~A}$ & 113.2 & $\mathrm{C} 5-\mathrm{C} 4-\mathrm{H} 4 \mathrm{~A}$ & 109.5 \\
\hline $\mathrm{C} 6-\mathrm{N} 2-\mathrm{H} 2 \mathrm{~B}$ & 109.5 & $\mathrm{C} 3-\mathrm{C} 4-\mathrm{H} 4 \mathrm{~B}$ & 109.5 \\
\hline $\mathrm{H} 2 \mathrm{~A}-\mathrm{N} 2-\mathrm{H} 2 \mathrm{~B}$ & 109.9 & $\mathrm{C} 5-\mathrm{C} 4-\mathrm{H} 4 \mathrm{~B}$ & 109.5 \\
\hline $\mathrm{N} 1-\mathrm{C} 1-\mathrm{C} 2$ & $108.11(15)$ & $\mathrm{H} 4 \mathrm{~A}-\mathrm{C} 4-\mathrm{H} 4 \mathrm{~B}$ & 108.1 \\
\hline $\mathrm{N} 1-\mathrm{C} 1-\mathrm{C} 6$ & $111.17(15)$ & $\mathrm{C} 4-\mathrm{C} 5-\mathrm{C} 6$ & $113.01(16)$ \\
\hline $\mathrm{C} 2-\mathrm{C} 1-\mathrm{C} 6$ & $112.27(15)$ & $\mathrm{C} 4-\mathrm{C} 5-\mathrm{H} 5 \mathrm{~A}$ & 109.0 \\
\hline $\mathrm{N} 1-\mathrm{C} 1-\mathrm{H} 1 \mathrm{D}$ & 108.4 & $\mathrm{C} 6-\mathrm{C} 5-\mathrm{H} 5 \mathrm{~A}$ & 109.0 \\
\hline $\mathrm{C} 2-\mathrm{C} 1-\mathrm{H} 1 \mathrm{D}$ & 108.4 & $\mathrm{C} 4-\mathrm{C} 5-\mathrm{H} 5 \mathrm{~B}$ & 109.0 \\
\hline $\mathrm{C} 6-\mathrm{C} 1-\mathrm{H} 1 \mathrm{D}$ & 108.4 & $\mathrm{C} 6-\mathrm{C} 5-\mathrm{H} 5 \mathrm{~B}$ & 109.0 \\
\hline $\mathrm{C} 3-\mathrm{C} 2-\mathrm{C} 1$ & $111.24(17)$ & $\mathrm{H} 5 \mathrm{~A}-\mathrm{C} 5-\mathrm{H} 5 \mathrm{~B}$ & 107.8 \\
\hline $\mathrm{C} 3-\mathrm{C} 2-\mathrm{H} 2 \mathrm{C}$ & 109.4 & $\mathrm{~N} 2-\mathrm{C} 6-\mathrm{C} 1$ & $110.14(15)$ \\
\hline $\mathrm{C} 1-\mathrm{C} 2-\mathrm{H} 2 \mathrm{C}$ & 109.4 & $\mathrm{~N} 2-\mathrm{C} 6-\mathrm{C} 5$ & $108.79(15)$ \\
\hline $\mathrm{C} 3-\mathrm{C} 2-\mathrm{H} 2 \mathrm{D}$ & 109.4 & $\mathrm{C} 1-\mathrm{C} 6-\mathrm{C} 5$ & $110.16(15)$ \\
\hline $\mathrm{C} 1-\mathrm{C} 2-\mathrm{H} 2 \mathrm{D}$ & 109.4 & $\mathrm{~N} 2-\mathrm{C} 6-\mathrm{H} 6 \mathrm{~A}$ & 109.2 \\
\hline $\mathrm{H} 2 \mathrm{C}-\mathrm{C} 2-\mathrm{H} 2 \mathrm{D}$ & 108.0 & $\mathrm{C} 1-\mathrm{C} 6-\mathrm{H} 6 \mathrm{~A}$ & 109.2 \\
\hline $\mathrm{C} 4-\mathrm{C} 3-\mathrm{C} 2$ & $111.09(17)$ & $\mathrm{C} 5-\mathrm{C} 6-\mathrm{H} 6 \mathrm{~A}$ & 109.2 \\
\hline $\mathrm{C} 4-\mathrm{C} 3-\mathrm{H} 3 \mathrm{~A}$ & 109.4 & $\mathrm{~N} 3-\mathrm{C} 7-\mathrm{S} 1$ & $178.2(2)$ \\
\hline $\mathrm{N} 1-\mathrm{C} 1-\mathrm{C} 2-\mathrm{C} 3$ & $178.41(15)$ & $\mathrm{C} 2-\mathrm{C} 1-\mathrm{C} 6-\mathrm{N} 2$ & $-173.39(15)$ \\
\hline $\mathrm{C} 6-\mathrm{C} 1-\mathrm{C} 2-\mathrm{C} 3$ & $55.4(2)$ & $\mathrm{N} 1-\mathrm{C} 1-\mathrm{C} 6-\mathrm{C} 5$ & $-174.63(15)$ \\
\hline $\mathrm{C} 1-\mathrm{C} 2-\mathrm{C} 3-\mathrm{C} 4$ & $-56.1(2)$ & $\mathrm{C} 2-\mathrm{C} 1-\mathrm{C} 6-\mathrm{C} 5$ & $-53.4(2)$ \\
\hline $\mathrm{C} 2-\mathrm{C} 3-\mathrm{C} 4-\mathrm{C} 5$ & $55.6(2)$ & $\mathrm{C} 4-\mathrm{C} 5-\mathrm{C} 6-\mathrm{N} 2$ & $174.46(16)$ \\
\hline $\mathrm{C} 3-\mathrm{C} 4-\mathrm{C} 5-\mathrm{C} 6$ & $-55.2(2)$ & $\mathrm{C} 4-\mathrm{C} 5-\mathrm{C} 6-\mathrm{C} 1$ & $53.6(2)$ \\
\hline $\mathrm{N} 1-\mathrm{C} 1-\mathrm{C} 6-\mathrm{N} 2$ & $65.36(19)$ & & \\
\hline
\end{tabular}

Hydrogen-bond geometry $\left(\AA,{ }^{\circ}\right)$

\begin{tabular}{lllll}
\hline$D-\mathrm{H} \cdots A$ & $D-\mathrm{H}$ & $\mathrm{H} \cdots A$ & $D \cdots A$ & $D-\mathrm{H} \cdots A$ \\
\hline $\mathrm{N} 1-\mathrm{H} 1 A \cdots \mathrm{S} 1^{\mathrm{i}}$ & 0.87 & 2.53 & $3.3914(19)$ & 172 \\
$\mathrm{~N} 1-\mathrm{H} 1 B \cdots \mathrm{N} 3^{\mathrm{ii}}$ & 0.82 & 2.10 & $2.895(3)$ & 166 \\
$\mathrm{~N} 1-\mathrm{H} 1 C \cdots \mathrm{N} 2^{\mathrm{iii}}$ & 1.01 & 1.83 & $2.841(2)$ & 175 \\
$\mathrm{~N} 2-\mathrm{H} 2 A \cdots \mathrm{N} 3^{\text {ii }}$ & 0.99 & 2.31 & $3.231(3)$ & 155 \\
$\mathrm{~N} 2-\mathrm{H} 2 B \cdots \mathrm{S} 1$ & 0.97 & 2.81 & $3.681(2)$ & 149 \\
\hline
\end{tabular}

Symmetry codes: (i) $x+1, y, z$; (ii) $-x+1,-y+1,-z$; (iii) $x+1 / 2,-y+1 / 2,-z$. 